\title{
Mortalidad juvenil, marginación social e inequidad en salud en México
}

\author{
Youth mortality, social marginalization and health inequity \\ in Mexico
}

${ }^{1}$ Centro de Estudios en Salud, Población y Desarrollo Humano, Departamento de Ciencias Sociales, Centro Universitario de Ciencias de la Salud, Universidad de Guadalajara. Sierra Mojada $950 \mathrm{Col}$. Independencia. 44340 Guadalajara México. ggonzal@cencar.udg.mx

\begin{abstract}
This study seeks to determine the differences in youth mortality in Mexico based on selected causes by sex and extreme levels of municipal marginalization in two triennia (2004-2006 and 2015-2017) and to establish a relationship between the differences found, the social environment and the availability of health resources. Using official data, years of life lost (YLL) between 0 and 85 years old and YLL for the 15-29-year-old age group were calculated for 15 of the main causes of death in Mexico in both triennia; the YLL was calculated for municipalities grouped into two categories: high and very high marginalization (HaVHMA) and low and very low marginalization (LaVLMA). Violent deaths (especially homicides) are the main causes of death in young women and men throughout Mexico, regardless of the level of marginalization, and increased from the first to second triennia studied. Men aged 15 to 29 years in HaVHMA municipalities had an excess YLL compared to those in LaVLMA municipalities in 13 of the 15 causes analyzed for 2004-2006 and in all causes for 2015-2017; for women, excess was observed for 13 of 15 causes in each triennium. These findings reflect the unfair disadvantages to which young people are exposed in HaVHMA municipalities.
\end{abstract}

Key words Mortality, Social marginalization, Health equity, Adolescents, Mexico
Resumen Este estudio busca determinar las diferencias en la mortalidad juvenil en México según causas seleccionadas, por sexo y niveles de marginación municipal extremos en dos trienios (20042006 y 2015-2017) y establecer una relación entre las diferencias encontradas, el entorno social y la disponibilidad de recursos de salud. Utilizando datos oficiales, se calcularon los Años de esperanza de vida perdidos (AEVP) entre 0 y 85 años $-y$ los AEVP por el grupo de edad de 15-29 años-para 15 de las principales causas de muerte en México en ambos trienios; los AEVP se calcularon por municipios agrupados en dos categorías: alta $y$ muy alta marginación (AyMAM) y baja y muy baja (ByMBM). Las muertes violentas (especialmente los homicidios) son las principales causas de muerte en mujeres y hombres jóvenes en todo México, independientemente del nivel de marginación, y aumentaron entre los dos trienios estudiados; los hombres de 15 a 29 años en municipios con AyMAM tuvieron un exceso de AEVP en comparación con aquellos en municipios con ByMBM en trece de las 15 causas analizadas en 2004-2006 $y$ en todas las causas en 2015-2017, mientras que para las mujeres la cifra fue de trece de 15 en cada trienio. Esto refleja las desventajas injustas a las que están expuestos los jóvenes en los municipios de AyMAM.

Palabras clave Mortalidad, Marginación social, Equidad en salud, Adolescentes, México 


\section{Introducción}

Las notables desigualdades socioeconómicas que subyacen en la gran mayoría de los países latinoamericanos, en especial en México ${ }^{1}$, son responsables en buena medida de las evidentes disparidades en los indicadores de salud que aún subsisten al interior de cada uno de ellos; un estudio reciente ha puesto de manifiesto las diferencias halladas en la esperanza de vida al nacer dentro de seis grandes urbes latinoamericanas (Ciudad de México incluida), debidas fundamentalmente a las desigualdades socioespaciales -entre ellas el estatus socioeconómico y la educación- identificadas en dichas ciudades $^{2}$.

Así, desde diferentes perspectivas metodológicas diversos estudios coinciden en revelar los grandes contrastes sociales existentes tanto en México como en otros países y como esto ha repercutido en los indicadores de mortalidad, morbilidad o de atención a la salud, identificando evidentes desventajas en la población más marginada y marcando una clara inequidad en el acceso a los servicios de salud, que perjudica a los sectores más desfavorecidos de la sociedad ${ }^{3-6}$.

En el contexto mexicano los estudios dirigidos a analizar la relación entre marginación y mortalidad juvenil han sido escasos; en general, se han centrado más en examinar el impacto de la marginación y la pobreza en algunos aspectos de la salud de adolescentes y jóvenes ${ }^{7,8}$-la fecundidad adolescente, por ejemplo9- que en el análisis de la mortalidad ${ }^{10}$. En un país con 32 millones de jóvenes entre 15 y 29 años, estudiar estos aspectos parece necesario ${ }^{11}$. La juventud es una etapa crítica en la que las desigualdades sociales pueden ensancharse o reducirse y obviamente tener un fuerte impacto en la salud juvenil; las trayectorias de vida ${ }^{12}$ muchas veces se entrecruzan y quedan obstruidas por un conjunto de circunstancias sociales que impiden que el joven encuentre las condiciones adecuadas para su desarrollo ${ }^{1}$, lo cual impacta en su salud y en su proyecto de vida.

En México, una forma de aproximación al estudio de las desigualdades sociales desde una perspectiva socioespacial lo constituye el análisis de la marginación, tanto a nivel estatal como municipal; el Consejo Nacional de Población (CONAPO) ${ }^{13}$ ha definido a la marginación como un fenómeno estructural de múltiples dimensiones que lleva implícito un desigual acceso de la población al desarrollo socioeconómico, y a la exclusión en el disfrute de sus beneficios ${ }^{14}$. En tal sentido, parecería lógico esperar que la existencia de importantes zonas geográficas del país altamente marginadas, vulnerables y, por ende, con elevados niveles de carencias de todo tipo se viera reflejado en la presencia de notorias inequidades sanitarias, esto es, diferencias en salud sistemáticas, evitables y marcadamente injustas entre las distintas áreas del país ${ }^{15}$.

A su vez, en las dos últimas décadas ha habido cambios significativos en el sistema de salud (como la implementación del llamado "seguro popular" para atender a la población sin seguridad social $)^{16}$, a la par de una intensificación del modelo neoliberal que ha marcado los últimos años del escenario económico y social del país, reflejado en la creciente desigualdad en el ingreso - de las más altas a nivel mundial ${ }^{17}-\mathrm{y}$ en el caso específico de la salud, en el debilitamiento de los servicios públi$\cos ^{16}$; este entorno sin dudas avala la importancia de comparar la mortalidad juvenil en dos momentos en el tiempo.

En tal sentido, el presente estudio pretende determinar las diferencias existentes en el comportamiento de la mortalidad juvenil en México según causas seleccionadas, por sexo y niveles extremos de marginación municipal en dos trienios (2004-2006 y 2015-2017) y establecer una posible relación entre las diferencias halladas, el entorno social y la disponibilidad de recursos y servicios en salud.

\section{Métodos}

Este es un estudio observacional, de carácter transversal y descriptivo, a partir de fuentes secundarias de información. Los datos sobre mortalidad y población correspondientes a los trienios analizados - tanto a nivel nacional como municipal- se obtuvieron de las bases de datos oficiales: de mortalidad de la Dirección General de Información en Salud de la Secretaría de Salud (DGIS) $)^{18}$, y de población, del CONAPO ${ }^{19}$. En particular, las causas de muerte seleccionadas -la mayoría de ellas consideradas entre las principales causas de muerte tanto para la población general como para la población juvenil ${ }^{18}$ - se clasificaron de acuerdo con la Clasificación Internacional de Enfermedades (CIE-10): homicidios (X85-Y09, Y87.1), accidentes de tráfico de vehículo motor (ATVM) [V02-V04 (.1, .9), V09.2-V09.3, V09.9, V12-V14 (.3-.9), V19.4-V19.6,V20-V28 (.3- .9), V29-V79 (.4-.9), V80.3-V80.5, V81.1, V82.1, V83-V86(.0-.3), V87.0-V87.8, V89.2, V89.9], suicidio (X60-X84, Y87,0), infecciones respiratorias agudas bajas (J10-J22), diabetes mellitus (E10-E14), enfermedades isquémicas del cora- 
zón (I20-I25), leucemia (C91-C95), VIH/SIDA (B20-B24), uso de alcohol (F10), enfermedades cerebrovasculares (I60-I69), desnutrición calórico-proteica (E43-E46), anemias (D50-D59), enfermedades infecciosas intestinales (A01-A09), cirrosis hepática (K70-K76) y enfermedad pulmonar obstructiva crónica (EPOC) (J40-J44).

A tenor con la definición del Instituto Mexicano de la Juventud ${ }^{20}$, en este estudio se reconoce como población juvenil aquella que se encuentra entre los 15 y 29 años, a partir de considerar los cambios demográficos -en particular el envejecimiento de la población- y sociales experimentados por la población mexicana en las últimas décadas.

Por otra parte, la información sobre el nivel de marginación municipal se obtuvo del CONA$\mathrm{PO}^{13}$ institución que ha construido desde $1990-\mathrm{y}$ actualizado cada cinco años- un índice de marginación a partir de nueve indicadores -analfabetismo, población sin educación primaria terminada, hacinamiento, viviendas particulares habitadas sin drenaje, sin energía eléctrica, sin agua entubada o con piso de tierra, población ocupada que recibe menos de dos salarios mínimos y población en localidades de menos de 5000 habitantes-, que reflejan las carencias en cuatro dimensiones (Educación, Vivienda, Ingresos Monetarios y Distribución de la Población); de acuerdo con el índice elaborado, CONAPO clasifica a los municipios -y también a los estados- en cinco categorías: muy alta, alta, media, baja y muy baja marginación. En esta investigación, por razones prácticas, se decidió trabajar solamente con dos categorías de marginación extrema, juntando los municipios de alta o muy alta marginación (AyMAM) y los de baja y muy baja marginación (ByMBM).

En 2015, México estaba dividido administrativamente en 32 estados y 2457 municipios (2454 en 2005), cuyo desarrollo económico y social es notoriamente diferente: de acuerdo con los criterios de CONAPO ${ }^{21}, 1100$ de estos municipios fueron clasificados en 2015 de AyMAM (1251 en 2005); el 95\% de los municipios de AyMAM en 2015 (1050) también estaban en esta condición en 2005; 515 de marginación media (502 en 2005) y 842 como de ByMBM, (701 en 2005). 674 de los municipios con ByMBM en 2015 (80\%) ya estaban en esta condición en 2005. Dado que una gran mayoría de los municipios han permanecido en su misma condición de marginación en ambos trienios, la comparación entre los dos momentos seleccionados parece pertinente.

Para el análisis de la mortalidad, se recurrió al método de Arriaga el cual ha sido empleado por diversos autores y aparece descrito en detalle en la literatura ${ }^{22-24}$. Este método permite calcular un índice que representa la diferencia entre el máximo posible de años que se pueden vivir entre dos edades y los años que realmente se viven; esto es, se calculan los años que no se viven -denominados por Arriaga como años de esperanza de vida perdidos (AEVP)-, en la práctica una medida del impacto potencial que tendría la reducción de la mortalidad por determinada causa sobre la esperanza de vida al nacer o a una edad en particu$\operatorname{lar}^{22}$.

Con los datos existentes sobre defunciones y población se construyeron tablas de mortalidad abreviadas para ambos sexos, tanto a nivel nacional como para las dos categorías de municipios construidas (AyMAM y ByMBM) en los trienios seleccionados, empleando para ello el programa EPIDAT v3.125. La elección de los trienios respondió a la disponibilidad de la información: 2015-2017 era el trienio más reciente para el que se disponía de datos que posibilitaban realizar el análisis previsto -la encuesta intercensal $2015^{26}$ permitía tener estimaciones confiables y actualizadas de la población municipal para el trienio seleccionado ${ }^{11}-$, y 2004-2006 era el trienio para el cual existía mayor información en la década anterior, por haberse realizado en 2005 el Conteo Nacional de Población y Viviendas, lo que permitiría ver posibles cambios en un lapso de tiempo razonable.

A partir de las tablas de mortalidad, y de acuerdo con el método mencionado se calcularon -mediante el programa EPIDAT v3.1 ${ }^{25}$ - tanto la esperanza de vida temporaria (EVT) entre 0 y 85 años como los AEVP entre las dos edades (en general, por cada causa seleccionada y por grupos de edad en cada trienio, para cada sexo) así como los AEVP entre las edades 15 y 85, 20 y 85 y 25 y 85 años, que en realidad constituyen un subconjunto del total de AEVP entre 0 y 85 años. Estos últimos pueden considerarse un indicador refinado -y a la vez robusto- tanto de la mortalidad juvenil por causas en los trienios analizados como del cambio en la mortalidad observado entre ambos momentos.

Además, en cada trienio se calcularon razones dividiendo los AEVP (total y de 15 a 29 años, por causa) de la agrupación de municipios de AyMAM entre los de ByMBM, con vistas a establecer la existencia de un posible exceso de mortalidad en los municipios clasificados como de AyMAM.

Los datos sobre recursos de salud para 2005 y 2016 se obtuvieron igualmente de bases de da- 
tos oficiales de la DGIS ${ }^{27}$, pero solo a nivel estatal, por lo que se ordenaron los 32 estados del país en cuatro cuartiles donde el cuartil I fue el que agrupó a los 8 estados con menor nivel de marginación y el IV, el que incluyó a los 8 estados con mayor nivel de marginación. Asimismo, la información sobre las características de los municipios -referidas a las fechas para las que existe información, 2005 y 2015- se obtuvo de las bases de datos del CONAPO ${ }^{24}$. Al igual que en el análisis de la mortalidad, se utilizaron razones para medir las diferencias entre los grupos extremos en cuanto a recursos de salud (nivel estatal) y en cuanto a las características de los municipios agrupados en las categorías AyMAM y ByMBM.

El protocolo de investigación "Mortalidad y Fecundidad en Jalisco y México", del que deriva este artículo, fue aprobado por el Centro de Estudios en Salud, Población y Desarrollo Humano de la Universidad de Guadalajara y registrado con la clave SyP- 2015-002. El proyecto se ajusta a la Declaración de Helsinki, así como a los lineamientos éticos expuestos en el Reglamento de la Ley General de Salud en materia de investigación para la salud de México, pues se consideró como "investigación sin riesgo", en tanto se analizan datos secundarios, empleándose técnicas y métodos de investigación documental -con datos protegidos- que no afectan a ningún individuo en particular.

\section{Resultados}

Las cifras expuestas en la Tabla 1 indican que la población mexicana se incrementó en casi 21 millones de habitantes entre 2004-2006 y 20152017, alcanzando los 122 millones de habitantes en este último trienio. De igual modo, aumentó en 21 millones la población residente en los municipios de la categoría ByMBM, por lo cual en el último trienio los habitantes de municipios en esta condición representaron alrededor de tres cuartas partes de la población total del país. Por el contrario, la población que habita en municipios de AyMAM disminuyó ligeramente entre ambos trienios, representando en 2015-2017 apenas el $14 \%$ del total de la población nacional, aunque el hecho de que más de 16 millones de personas vivan en municipios altamente marginados es sin dudas relevante.

La Tabla 1 también permite observar que mientras a nivel nacional -y sobre todo en el grupo de municipios de ByMBM- se aprecia un aumento en la EVT en ambos sexos (más marcado
Tabla 1. Esperanza de vida temporaria (EVT) entre 0 y 85 años, y cambio absoluto entre ambos trienios; población anual promedio y defunciones anuales promedio en cada trienio, por sexo. México, municipios de Alta y Muy Alta marginación y Baja y Muy Baja marginación, 2004-2006 y 2015-2017.

\begin{tabular}{|c|c|c|}
\hline & Hombres & Mujeres \\
\hline & \multicolumn{2}{|c|}{$\begin{array}{l}\text { Alta y Muy Alta } \\
\text { Marginación }\end{array}$} \\
\hline EVT 2004-2006 & 71,03 & 74,89 \\
\hline EVT 2015-2017 & 69,05 & 73,87 \\
\hline Cambio Absoluto & $-1,98$ & $-1,02$ \\
\hline $\begin{array}{l}\text { Población anual promedio } \\
(2004-2006)\end{array}$ & 8225543 & 8628305 \\
\hline $\begin{array}{l}\text { Defunciones anuales } \\
\text { promedio (2004-2006) }\end{array}$ & 45720 & 34806 \\
\hline $\begin{array}{l}\text { Población anual promedio } \\
(2015-2017)\end{array}$ & 8200253 & 8551283 \\
\hline \multirow{2}{*}{$\begin{array}{l}\text { Defunciones anuales } \\
\text { promedio (2015-2017) }\end{array}$} & 63285 & 50084 \\
\hline & \multicolumn{2}{|c|}{$\begin{array}{c}\text { Baja y Muy Baja } \\
\text { Marginación }\end{array}$} \\
\hline EVT 2004-2006 & 71,10 & 75,21 \\
\hline EVT 2015-2017 & 71,67 & 76,30 \\
\hline Cambio Absoluto & 0,57 & 1,09 \\
\hline $\begin{array}{l}\text { Población anual promedio } \\
(2004-2006)\end{array}$ & 35014849 & 36961508 \\
\hline $\begin{array}{l}\text { Defunciones anuales } \\
\text { promedio }(2004-2006)\end{array}$ & 187924 & 155503 \\
\hline $\begin{array}{l}\text { Población anual promedio } \\
(2015-2017)\end{array}$ & 45351704 & 47672067 \\
\hline \multirow{2}{*}{$\begin{array}{l}\text { Defunciones anuales } \\
\text { promedio (2015-2017) }\end{array}$} & 265873 & 212771 \\
\hline & \multicolumn{2}{|c|}{ México } \\
\hline EVT 2004-2006 & 71,07 & 75,18 \\
\hline EVT 2015-2017 & 71,26 & 75,81 \\
\hline Cambio Absoluto & 0,19 & 0,63 \\
\hline $\begin{array}{l}\text { Población anual promedio } \\
(2004-2006)\end{array}$ & 49328900 & 51991852 \\
\hline $\begin{array}{l}\text { Defunciones anuales } \\
\text { promedio (2004-2006) }\end{array}$ & 280913 & 227128 \\
\hline $\begin{array}{l}\text { Población anual promedio } \\
(2015-2017)\end{array}$ & 59640464 & 62625390 \\
\hline $\begin{array}{l}\text { Defunciones anuales } \\
\text { promedio }(2015-2017)\end{array}$ & 376428 & 299674 \\
\hline
\end{tabular}

en el femenino), en el grupo de municipios de AyMAM la EVT se redujo de forma importante entre ambos trienios: casi en dos años en el caso de los hombres y en un año para las mujeres.

Una caracterización socioeconómica de los municipios de México, de acuerdo con su nivel de 
marginación social, alrededor de 2005 y 2015, y haciendo énfasis, en la medida de lo posible en la situación de los jóvenes, se presenta en la Tabla 2.

En primer lugar, se observó que, en 2015, la proporción de población juvenil fue algo mayor en los municipios con AyMAM que en los municipios con ByMBM. Asimismo, la proporción de población juvenil sin seguro social en el grupo de municipios de AyMAM -alrededor del 90\%- fue $80 \%$ mayor a la de los municipios con ByMBM en ambos momentos. El porcentaje de mujeres de 15 a 19 años sin primaria terminada en los municipios con AyMAM más que triplicó el del grupo de ByMBM en los dos años analizados y la proporción de jóvenes que ya a esta edad están casadas o unidas se incrementó en el grupo de AyMAM y se redujo en el de ByMBM, aumentando la diferencia entre ellos.

En sentido general los datos reflejan -en ambos momentos- perfiles sociales contrastantes para ambos grupos de municipios, mantenidos (o incluso acrecentados) en el tiempo: mientras que la población en los municipios de AyMAM vive en su mayoría en localidades pequeñas (muchas de ellas de carácter rural) y en viviendas que carecen de servicios básicos como agua entubada; en donde más de las dos terceras partes de las viviendas tienen muy bajos ingresos y más de la tercera parte de los habitantes de 15 años o más tiene educación primaria incompleta, en los municipios de ByMBM las características que presenta la población residente evidencian en general mejores condiciones de vida.

En la Tabla 3 se puede apreciar que en 20042006 las causas que provocaron más AEVP en los municipios de AyMAM para la población masculina en general fueron cirrosis, diabetes $y$ homicidio (por ese orden), mientras que para los jóvenes de 15 a 29 años fueron homicidio, ATVM y suicidio. En el caso de los municipios de ByMBM, para el total de hombres las causas que provocaron más AEVP fueron diabetes, enfermedades isquémicas del corazón y cirrosis, mientras para el grupo de 15 a 29 las más relevantes fueron ATVM, homicidio y suicidio.

En 2015-2017, se mantiene en sentido general este comportamiento, aunque con algunos intercambios de posiciones: en los municipios de AyMAM diabetes, cirrosis y homicidio (en ese orden) fueron las principales causantes de AEVP para los hombres en general, pero para los jóvenes de 15 a 29 años fueron homicidio, ATVM y suicidio. En los municipios con ByMBM, para

Tabla 2. Características socioeconómicas de la población (en porcentaje) según nivel de marginación y Razón municipios de Alta y Muy Alta marginación / municipios de Baja y Muy Baja marginación. México, 2005 y 2015.

\begin{tabular}{|c|c|c|c|}
\hline 2005 & $\begin{array}{c}\text { Alto y Muy } \\
\text { Alto (AA) }\end{array}$ & $\begin{array}{c}\text { Bajo y muy } \\
\text { Bajo (BB) }\end{array}$ & $\begin{array}{l}\text { Razón } \\
\text { AA/BB }\end{array}$ \\
\hline Porcentaje de jóvenes entre 15 y 29 años de la población total & 25,75 & 27,44 & 0,94 \\
\hline Porcentaje de jóvenes entre 15 y 29 años sin Seguridad Social & 91,42 & 50,37 & 1,81 \\
\hline Porcentaje de mujeres entre 15 y 19 años sin primaria terminada & 9,51 & 2,62 & 3,63 \\
\hline Porcentaje de mujeres entre 15 y 19 años casadas o unidas & 17,63 & 14,12 & 1,25 \\
\hline Porcentaje de viviendas sin agua entubada & 31,03 & 4,58 & 6,77 \\
\hline Porcentaje de habitantes $>14$ años sin primaria terminada & 47,38 & 16,40 & 2,89 \\
\hline Porcentaje de viviendas con ingresos $<$ a 2 salarios mínimos ${ }^{\star}$ & 75,42 & 36,65 & 2,06 \\
\hline Porcentaje de población residente en localidades de $>$ de 5000 habitantes & 81,22 & 12,44 & 6,53 \\
\hline (2: & $\begin{array}{c}\text { Alto y Muy } \\
\text { Alto (AA) }\end{array}$ & $\begin{array}{c}\text { Bajo y muy } \\
\text { Bajo (BB) }\end{array}$ & $\begin{array}{l}\text { Razón } \\
\text { AA/BB }\end{array}$ \\
\hline Porcentaje de jóvenes entre 15 y 29 años de la población total & 28,24 & 25,49 & 1,11 \\
\hline Porcentaje de jóvenes entre 15 y 29 años sin Seguridad Social & 91,56 & 50,41 & 1,82 \\
\hline Porcentaje de mujeres entre 15 y 19 años sin primaria terminada & 6,03 & 1,88 & 3,21 \\
\hline Porcentaje de mujeres entre 15 y 19 años casadas o unidas & 19,11 & 13,53 & 1,41 \\
\hline Porcentaje de viviendas sin agua entubada & 17,35 & 2,85 & 6,09 \\
\hline Porcentaje de habitantes $>14$ años sin primaria terminada & 36,95 & 12,03 & 3,07 \\
\hline Porcentaje de viviendas con ingresos $<$ a 2 salarios mínimos ${ }^{\star}$ & 65,88 & 32,60 & 2,02 \\
\hline Porcentaje de población residente en localidades de $<$ de 5000 habitantes & 80,65 & 14,91 & 5,41 \\
\hline
\end{tabular}

*2 salarios mínimos $=8$ USD diarios aproximadamente en ambos años.

Fuente: Consejo Nacional de Población (CONAPO). Índice de marginación por entidad federativa y municipio $1990-2015$. Disponible en: https://www.gob.mx/conapo/documentos/indice-de-marginacion-por-entidad-federativa-y-municipio-2015 [acceso $2020 \mathrm{Ag} 15]$. 


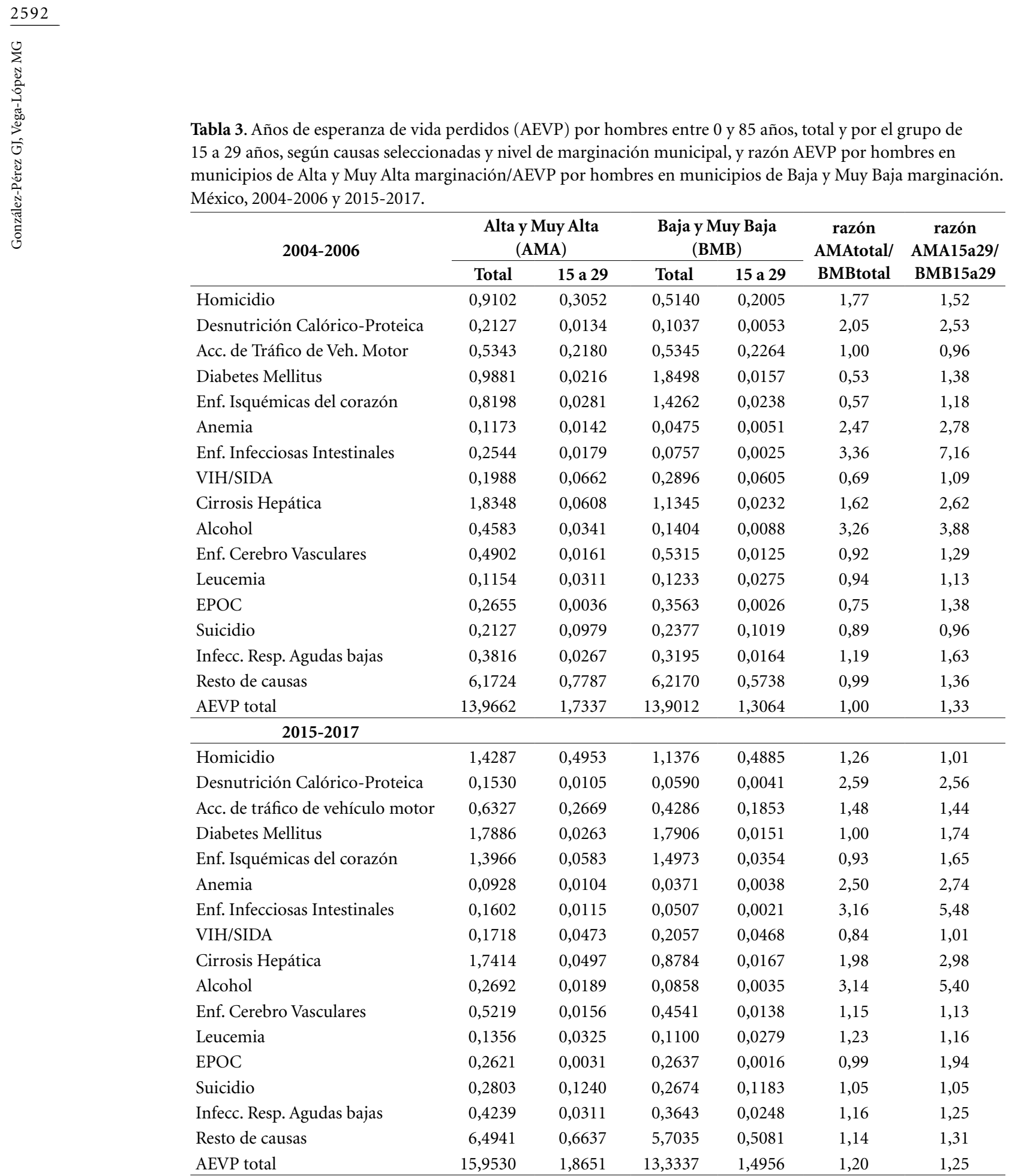

Fuente: Elaboración propia a partir de resultados del estudio.

el total de la población masculina las causas que provocaron más AEVP fueron diabetes, enfermedades isquémicas del corazón y homicidio, mientras para el grupo de 15 a 29 las más relevantes fueron homicidio, ATVM y suicidio.
En el grupo de municipios con AyMAM en el grupo de 15 a 29 años se observó un aumento de los AEVP en siete de las 15 causas: los AEVP por enfermedades isquémicas del corazón se duplicaron entre ambos trienios, mientras que los 
AEVP causados por homicidios, suicidios y diabetes también tuvieron un alza importante. En los municipios de ByMBM, en el grupo de 15 a 29 años disminuyeron los AEVP en nueve causas (cirrosis, diabetes y ATVM entre ellas), siendo homicidios la causa en la que más se incrementaron los AEVP, más del doble en 2015-2017 de los calculados para 2004-2006.

Al comparar en el grupo de 15 a 29 años el número de AEVP por causas entre los municipios con AyMAM y aquellos con ByMBM, en trece de 15 causas se observó un exceso de AEVP en el grupo de municipios con AyMAM; además de las enfermedades infecciosas intestinales -que más que septuplican los AEVP en el grupo de municipios de ByMBM- la desnutrición calórico-proteica, anemia y cirrosis también presentan un importante exceso de AEVP. Solo suicidio y ATVM presentan razones inferiores a uno, es decir que el número de AEVP fue mayor en el grupo de municipios de ByMBM.

Al realizar una comparación similar en el trienio 2015-2017, las razones calculadas evidencian que para el grupo de 15 a 29 años, en todas las 15 causas se observó un exceso de AEVP en el grupo de municipios con AyMAM con relación a los de ByMBM. Además de las Enfermedades Infecciosas Intestinales y el Uso de alcohol -que más que quintuplican los AEVP en el grupo de municipios de ByMBM- la desnutrición calórico-proteica y la anemia también presentan un importante exceso de AEVP. La diabetes y las enfermedades isquémicas del corazón también presentan razones relativamente altas, no así los homicidios.

Además, en nueve de las 15 causas se apreció en 2015-2017 un mayor exceso de AEVP en los municipios con AyMAM en relación con el grupo de ByMBM al compararlo con las razones calculadas para 2004-2006. Por ejemplo, el exceso de AEVP por diabetes en 2004-2006 fue de 44\% y pasó a ser de 74\% en 2015-2017. Además, en ambos trienios el número total de AEVP por los jóvenes de municipios con AyMAM fue mayor al observado en los municipios de ByMBM (33\% mayor en 2004-2006 y 25\% en 2015-2017).

En el caso de las mujeres (Tabla 4) el análisis de los AEVP según nivel de marginación es en buena medida similar. Las cifras evidencian que mientras las causas que provocaron más AEVP en 2004-2006 en los municipios de AyMAM para la población femenina en general fueron diabetes, enfermedades isquémicas del corazón y cirrosis (en esa posición), para las jóvenes de 15 a 29 años fueron ATVM, homicidio y suicidio. En el caso de los municipios de ByMBM, para el total de las mujeres las causas que provocaron más AEVP fueron diabetes, enfermedades isquémicas del corazón y enfermedades cerebrovasculares, mientras para el grupo de 15 a 29 las más relevantes fueron ATVM, homicidio y suicidio.

En 2015-2017, se observa un comportamiento parecido, aunque también con algunas posiciones cambiantes: en los municipios de AyMAM diabetes, enfermedades isquémicas del corazón y cirrosis (en ese orden) provocaron el mayor número de AEVP para el total de mujeres -la diabetes por si sola provocó la pérdida de más de 2 años de esperanza de vida-, pero para las jóvenes de 15 a 29 años fueron homicidio, suicidio y ATVM. En los municipios con ByMBM, para el total de la población femenina las causas que provocaron más AEVP fueron diabetes, enfermedades isquémicas del corazón y enfermedades cerebrovasculares, mientras para el grupo de 15 a 29 las más relevantes fueron homicidio, ATVM y suicidio.

En el grupo de municipios con AyMAM, para las jóvenes de 15 a 29 años se observó un aumento de los AEVP en cinco causas: los AEVP por homicidios casi se duplicaron entre ambos trienios, mientras que los AEVP causados por suicidios, enfermedades isquémicas del corazón y diabetes también se elevaron de forma notable. En los municipios con ByMBM, en el grupo de 15 a 29 años decrecieron los AEVP en once causas (diabetes y ATVM entre ellas), siendo homicidios la causa en la que más se elevaron los AEVP, más del doble en 2015-2017 de los calculados para 2004-2006.

Las razones calculadas para comparar el número de AEVP por causas entre los municipios con AyMAM y aquellos con ByMBM, muestran que en 2004-2006 para las jóvenes de 15 a 29 años, en trece de 15 causas se apreció un exceso de AEVP en el grupo de municipios con AyMAM; además de las enfermedades infecciosas intestinales -que presentan casi ocho veces más AEVP que en el grupo de municipios de ByMBM- la desnutrición calórico-proteica, anemia y cirrosis también presentan un importante exceso, pues triplican el número de AEVP observado en los municipios de ByMBM. Solo homicidio y ATVM presentan razones inferiores a uno, es decir que el número de AEVP fue mayor en el grupo de municipios de ByMBM.

La misma comparación, pero para el trienio 2015-2017, pone de manifiesto que para las jóvenes, en trece de las 15 causas se observó un exceso de AEVP en el grupo de municipios con AyMAM con relación a los de ByMBM, siendo 


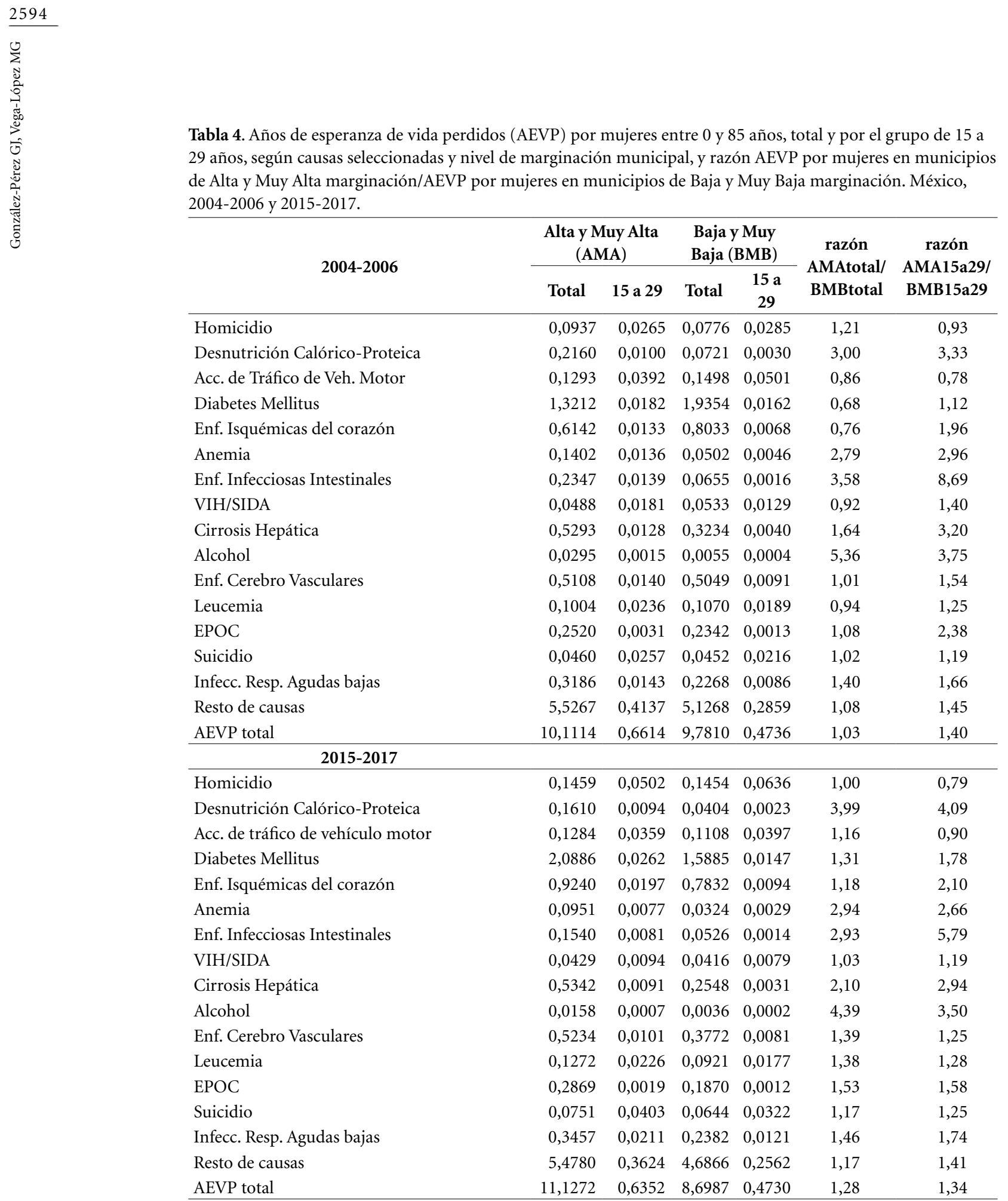

Fuente: Elaboración propia a partir de resultados del estudio.

las enfermedades infecciosas intestinales, el uso de alcohol y la desnutrición calórico-proteica las causas que presentan las razones más altas.

Asimismo, en seis de las 15 causas se puede advertir en 2015-2017 un mayor exceso de AEVP en los municipios con AyMAM con relación al grupo de ByMBM al compararlo con las razones calculadas para 2004-2006. Es el caso de la diabetes, que tenía un exceso de AEVP en 2004-2006 de $12 \%$ y se incrementó a $78 \%$ en 2015-2017. Finalmente, el número total de AEVP por mujeres del grupo de 15 a 29 años en municipios con Ay- 
MAM fue mayor al observado en los municipios con ByMBM en los dos trienios (40\% mayor en 2004-2006 y 34\% en 2015-2017).

Por último, la Tabla 5 brinda elementos para documentar como se distribuyen los recursos humanos y materiales y los servicios de salud en el país, de acuerdo con el grado de marginación estatal, para los años centrales de los dos trienios. Los 32 estados se ordenaron en cuartiles, siendo el cuartil I el que agrupa a los 8 estados con menor marginación (incluye estados como la Ciudad de México, Nuevo León o Baja California, los mismos estados coinciden en ambos años) y el cuartil IV, el que agrupa a los 8 estados con el mayor índice de marginación (entre ellos Chiapas, Oaxaca y Guerrero, con solo un cambio entre los dos años estudiados).

Aunque varios de los indicadores para los estados con mayor marginación (cuartil IV) en 2016 evidencian una mejoría con respecto a 2005 -incremento al gasto público en salud, en camas hospitalarias, médicos y personal de enfermería titulado, entre otros- siguen existiendo importantes déficits en cuanto a médicos especialistas y a enfermeros por médico, y subsisten notables disparidades entre los estados de marginación extrema.

En tal sentido, en 2016 el gasto público en salud en los estados en el cuartil I todavía fue $68 \%$ mayor que en el cuartil IV y esto se refleja en otros indicadores, que se comportan mucho mejor en los estados menos marginados que en aquellos con más alta marginación.-

\section{Discusión}

Aun cuando en la actualidad las personas están viviendo más años en todo el mundo ${ }^{28}$, “....sigue habiendo un considerable desfase entre ricos y pobres: las personas de los países de altos ingresos siguen teniendo muchas más probabilidades de vivir más años que las personas de países de bajos ingresos" 29 .

Ese desfase se hace más evidente al interior de naciones que, como México, presentan grandes disparidades socioeconómicas; en tal sentido, el presente estudio ha puesto de manifiesto aspectos relevantes como los citados a continuación.

La EVT entre 0 y 85 años para ambos sexos fue más elevada en los dos trienios estudiados en el grupo de municipios con ByMBM que en el de AyMAM; en este último grupo, la EVT se redujo para ambos sexos (sobre todo los hombres) entre 2004-2006 y 2015-2017.

El número total de AEVP a causa de la mortalidad juvenil, en ambos sexos y en ambos trienios es claramente mayor -al menos $25 \%$ más- en los municipios con AyMAM que en los de ByMBM (una proporción mayor en cada caso que la de la población general).

Tabla 5. Indicadores de recursos humanos y materiales en salud según nivel de marginación estatal y Razón Cuartil I (menor marginación/Cuartil IV (mayor marginación)). México, 2005 y 2016.

\begin{tabular}{lccccc}
\hline \multicolumn{1}{c}{ Indicadores 2005 } & I & II & III & IV & $\begin{array}{c}\text { Razón } \\
\text { I/IV }\end{array}$ \\
\hline Camas hospitalarias censables por 10000 hab. & 4,27 & 2,86 & 2,88 & 2,85 & 1,50 \\
Médicos titulados en contacto con pacientes por 10000 hab. & 5,65 & 3,06 & 4,41 & 3,79 & 1,49 \\
Médicos especialistas por cada médico general & 2,92 & 1,21 & 0,87 & 0,71 & 4,11 \\
Enfermeros titulados por cada 10000hab. & 6,17 & 3,26 & 4,43 & 3,80 & 1,62 \\
Enfermeros por médico & 1,09 & 1,06 & 1,01 & 1,00 & 1,09 \\
Gasto público en salud per cápita (en pesos mex.)* & 3479 & 1717 & 2007 & 1660 & 2,10 \\
\hline \multicolumn{1}{c}{ Indicadores 2016 } & I & II & III & IV & Razón \\
& & & & & I/IV \\
\hline Camas hospitalarias censables por 10000 hab. & 3,89 & 2,77 & 3,37 & 3,09 & 1,26 \\
Médicos titulados en contacto con pacientes por 10000 hab. & 7,22 & 5,79 & 7,11 & 6,71 & 1,08 \\
Médicos especialistas por cada médico general & 2,48 & 1,17 & 0,81 & 0,77 & 3,22 \\
Enfermeros titulados por cada 10000hab. & 8,05 & 6,56 & 8,01 & 6,48 & 1,24 \\
Enfermeros por médico & 1,13 & 1,11 & 1,04 & 0,97 & 1,16 \\
Gasto público en salud per cápita (en pesos mex.)* & 6496 & 4585 & 4327 & 3861 & 1,68 \\
\hline
\end{tabular}

${ }^{*} 1$ USD $=11$ pesos mexicanos en 2005/1USD=18 pesos mexicanos en 2016 aproximadamente. 
Los hombres de 15 a 29 años en los municipios con AyMAM tuvieron un exceso de AEVP con relación a los de municipios con ByMBM en trece de las 15 causas analizadas en 2004-2006 y en todas las causas en 2015-2017, mientras que para las mujeres la cifra fue trece de 15 en cada trienio.

Comparado con el trienio anterior, en 20152017 en nueve de las 15 causas se incrementó el exceso del número de AEVP por los hombres de 15 a 29 años en municipios con AyMAM con relación a los de ByMBM -seis en el caso de las jóvenes.

En los municipios con AyMAM, la proporción de jóvenes entre 15 y 29 años se ha incrementado y es mayor que en los municipios con ByMBM, pero la cobertura de la seguridad social a esta población es $80 \%$ menor y su rezago educativo es mayor.

En general, hay un notorio contraste entre las características socioeconómicas y obviamente el perfil de la población -en particular la juvenil- de los municipios de ByMBM con respecto al grupo de municipios con AyMAM, que pone a estos últimos en una clara desventaja social; y de igual modo el gasto público en salud y los recursos disponibles en salud -médicos especialistas, enfermeras, camas- a pesar de su mejoría, continúan siendo mucho más reducidos en los estados más marginados, donde más se necesitan.

Los hallazgos antes mencionados apuntan decididamente a la existencia no solo de una mayor mortalidad juvenil en los municipios de AyMAM al compararla con los municipios de ByMBM, sino también al incremento de la brecha que separa en cuanto a la mortalidad a los jóvenes de ambos conjuntos de municipios, una situación que se manifiesta de manera más marcada que en la población en general.

Es en este escenario que algunos resultados de esta investigación deben ser evaluados con más detenimiento. En primer lugar, es importante destacar que las muertes violentas (homicidios, suicidios, y ATVM), causas esencialmente evitables, son las principales causas de muerte en mujeres y hombres jóvenes de todo México -independientemente del nivel de marginación- y se incrementaron sustancialmente entre ambos trienios estudiados; en especial, los homicidios provocaron entre los hombres la pérdida de casi medio año de esperanza de vida en cada grupo de municipios en 2015-2017 y la reducción del exceso de mortalidad observado en los municipios con AyMAM entre los dos quinquenios -o incluso la mayor sobremortalidad de las mujeres que residen en municipios de ByMBM- parece estar asociada a un proceso de "urbanización de la violencia" ${ }^{\text {"30,31, }}$, resultado de la lucha de los carteles del narcotráfico por el control de plazas en las ciudades del país, lo cual ha provocado el aumento de la mortalidad por homicidios en municipios de ByMBM.

Por otra parte, la falta de empleo o la precarización del mismo, la carencia de plazas suficientes para acceder a la educación superior, $o$ la convivencia cotidiana con conductas violentas, son factores asociados a que los jóvenes sean víctimas de homicidio, una situación que se acentúa en las comunidades más marginadas, en las cuales es más probable que un joven ni estudie ni trabaje ${ }^{12,31}$; muchos de estos aspectos también están relacionados con el aumento de la mortalidad por suicidio en los jóvenes de ambos sexos, más marcado en los residentes en municipios con AyMAM.

Otras causas evitables -como las enfermedades infecciosas intestinales, la desnutrición calórico-proteica o la anemia-si bien han disminuido su mortalidad en los municipios de AyMAM, aún reflejan un exceso importante al comparar los AEVP con el conjunto de municipios de ByMBM -exceso este que en algunas causas incluso se ha incrementado (dado que el descenso por estas causas en el grupo de municipios de ByMBM ha sido mayor); los problemas de desnutrición, mucho más prevalentes en las comunidades más marginadas ${ }^{32}$ asociados a la carencia de recursos económicos para revertir dicha situación y a las desventajas documentadas previamente en cuanto a educación y acceso a los servicios de salud para los municipios con AyMAM ${ }^{5}$, hacen que este exceso de mortalidad sea injusto e inaceptable, en tanto en la actualidad estas causas no deberían en la práctica provocar defunciones- $y$ refleja la vulnerabilidad de la población juvenil que vive en zonas altamente marginadas.

A diferencia de las causas potencialmente evitables, la mortalidad por enfermedades crónico-degenerativas -diabetes, enfermedades isquémicas del corazón, sobre todo- se ha incrementado en los municipios con AyMAM, y coincidentemente, se ha reducido (o no ha aumentado tanto) en los municipios de ByMBM, lo que explica en 2015-2017 una sobremortalidad que casi no existía en 2004-2006. Aun cuando estas causas no son en la actualidad de las más relevantes en la mortalidad juvenil, su mayor crecimiento en las áreas de AyMAM evidencia, sobre todo, una mayor velocidad en el aumento del sobrepeso y la obesidad en la población más mar- 
ginada $^{32}$ y en particular, en la población adolescente rural (que vive mayormente en municipios de AyMAM) $)^{33}$. Se ha documentado el impacto del consumo de comida chatarra en el cambio de los hábitos alimenticios de niños y adolescentes en comunidades rurales marginadas del país ${ }^{8}$ y la prevalencia de dietas en las que predominan alimentos con alto contenido calórico ${ }^{7}$, pero que son más accesibles a las familias con escasos ingresos, como sucede en los municipios de AyMAM.

Una mención especial merece el caso de la leucemia: ha sido documentada la tendencia al alza de la mortalidad por cáncer -en especial leucemia- en adolescentes mexicanos entre 1990 y 2009 en estados de AyMAM $^{10}$; esta causa también presenta en mujeres y hombres jóvenes residentes en municipios de AyMAM cifras de AEVP mayores a los de los jóvenes que viven en municipios de ByMBM, exceso que se ha incrementado en el lapso analizado. Aun cuando la mejoría en la calidad de los registros haya podido influir en el incremento de las cifras en las áreas más marginadas, las desventajas socioeconómicas y de acceso a los servicios de salud -el seguro popular excluye la atención a la mayor parte de los padecimientos de alto costo, cuyo tratamiento lo tendría que pagar el paciente, y en particular no incluye el tratamiento de las leucemias en mayores de 18 años ${ }^{16,34}-$ sin dudas son un freno para lograr un tratamiento exitoso y aumentar la sobrevida de los jóvenes que padecen esta enfermedad.

Para enfrentar $-y$ tratar de revertir- esta situación inequitativa en términos sanitarios y dar respuesta a las crecientes demandas de la población juvenil se necesitarían profundos cambios estructurales que impulsen el desarrollo social y económico (empleos dignos, acceso a educación superior), el fortalecimiento de los sistemas de salud y de seguridad social -y la implementación de verdaderos sistemas de salud y protección social universales ${ }^{35}-\mathrm{y}$ que permitan reducir las desventajas injustas a las que están expuestos importantes núcleos poblacionales delpaís, en especial aquellos en condiciones de AyMAM. En tal sentido, las políticas sociales -que mayormente invisibilizan a la población juvenil, o en el mejor de los casos la consideran como receptora de acciones de carácter asistencial ${ }^{1}-$ deben convertir al joven en protagonista y brindarle los elementos necesarios para modificar su situación, más en aquellos casos que viven en condiciones de desventaja social.

El presente estudio puede tener algunas limitaciones, referidas sustancialmente a la recolección de datos de mortalidad. Es bien conocido que el nivel de subregistro de la mortalidad puede estar relacionado con las desigualdades socioeconómicas, siendo generalmente más elevado en los municipios más marginados; esto pudiera reflejarse en una subestimación de la mortalidad observada para los municipios con AyMAM, lo cual posiblemente acrecentaría el exceso de mortalidad de estos municipios con respecto a los de ByMBM. Aunque la cobertura y calidad de los registros de mortalidad en México ha sido reconocida por la Organización Panamericana de la Salud como satisfactoria ${ }^{36}$, se debe mirar con cierta cautela cualquier resultado extraído de las tablas de mortalidad, como la EVT o los AEVP, así como las comparaciones realizadas.

Por otra parte, aunque la ausencia en la selección de causas estudiadas de algunos tumores malignos podría considerarse una limitación del estudio, la inclusión de la leucemia -responsable de un tercio de las muertes por tumores malignos en la población juvenil en años recientes ${ }^{18}$ - puede atenuar esta situación.

A pesar de las limitaciones previamente expuestas, los resultados hallados permiten una aproximación razonable y novedosa al comportamiento reciente de la mortalidad juvenil por causas en México, y en particular permite evidenciar las disparidades existentes según nivel de marginación. El uso de un indicador como los AEVP agrega un valor estratégico al análisis de los cambios de la mortalidad juvenil por causas en años recientes y ofrece a quienes toman decisiones evidencias concretas de las inequidades sanitarias persistentes en el país. 


\section{Colaboradores}

GJ González-Pérez participó en el diseño y coordinación del estudio, recolección de datos, análisis estadístico, interpretación de los datos y la redacción del manuscrito. MG Vega-López participó en el diseño del estudio, recolección de datos el análisis e interpretación de los datos y la redacción del manuscrito. Todos los autores revisaron y aprobaron la versión final del artículo.

\section{Referencias}

1. Comisión Económica para América Latina y el Caribe (CEPAL). La matriz de la desigualdad social en América Latina. Santiago de Chile: Naciones Unidas; 2016.

2. Bilal U, Alazraqui M, Caiaffa T, Lopez-Olmedo N, Martinez-Folgar K, Miranda JJ, Rodriguez DA, Vives A, Diez-Roux AV. Inequalities in life expectancy in six large Latin American cities from the SALURBAL study: an ecological analysis. Lancet Planet Health 2019; 3: e503-e510.

3. Zurita B, Lozano R, Ramírez T, Torres JL. Desigualdad e Inequidad en Salud. En: Knaul F, Nigenda G. Caleidoscopio de la Salud. México: Fundación Mexicana para la Salud; 2003. p. 29-39.

4. González-Pérez GJ, Vega-López MG, Romero-Valle S, Vega-López A, Cabrera-Pivaral CE. Exclusión social e inequidad en salud en México: un análisis socioespacial. Rev Salud Publica 2008; 10(Supl. 1):15-28.

5. Barraza-Lloréns M, Panopoulou G, Díaz BY. Income -related inequalities and inequities in health and health care utilization in Mexico, 2000-2006. Rev Panam Salud Publica 2013; 33(2):122-130.

6. Guimarães RM. A teoria da equidade reversa se aplica na atenção primária à saúde? Evidências de 5.564 municípios brasileiros. Rev Panam Salud Publica 2018; 42:128.

7. Castañeda-Castaneira E, Ortiz-Pérez H, Robles-Pinto G, Molina-Frechero N. Consumo de alimentos chatarra y estado nutricio en escolares de la Ciudad de México. Rev Mex Pediatr 2016; 83(1):15-19.

8. Del Ángel-Pérez AL, Villagómez-Cortés JA. Alimentación, salud y pobreza en áreas marginadas urbanas: caso Veracruz-Boca del Río, Veracruz, México. Estud Soc 2014; 44:11-35.

9. Garcia-Saiso S, Gutiérrez JP, Pacheco Estrello P, Franco J, González E, Gutiérrez-Delgado C, Barriga B, Martínez-Valle A, Choperena-Aguilar DG, GonzálezGuzmán R, Laurell AC. Primer Informe sobre Desigualdades en Salud en México. Ciudad de México: Secretaría de Salud/(OPS); 2019.

10. Escamilla-Santiago RA, Narro-Robles J, Fajardo-Gutiérrez A, Rascón-Pacheco RA, López-Cervantes M. Tendencia de la mortalidad por cáncer en niños y adolescentes según grado de marginación en México (1990-2009). Salud Publica Mex 2012; 54:587-594.

11. Consejo Nacional de Población (CONAPO). Proyecciones de la Población de los Municipios de México, 2015-2030 [Internet]. [acceso 2020 Feb 27]. Disponible en: https://www.gob.mx/conapo/documentos/ proyecciones-de-la-poblacion-de-los-municipios-demexico-2015-2030.

12. Organización para la Cooperación y el Desarrollo Económicos. Panorama de la Educación 2014: Indicadores de la OCDE [Internet]. 2014 [acceso 2017 Oct 15]. Disponible en: https://tinyurl.com/yc2qathy.

13. Consejo Nacional de Población (CONAPO). Capítulo 1. Concepto y dimensiones de la marginación [Internet]. [acceso $2020 \mathrm{Feb} 25]$. Disponible en: http://www. conapo.gob.mx/work/models/CONAPO/Resource/1755/1/images/01Capitulo.pdf. 
14. Bel AC. Exclusión Social: origen y características. En Curso: "Formación específica en Compensación Educativa e Intercultural para Agentes Educativos" [Internet]. Murcia; 2002 [acceso 2017 Feb 24]. Disponible en: http://stepv.intersindical.org/enxarxats/nee/CE_exclusio.pdf.

15. Whitehead M. The concepts and principles of equity and health. Int J Health Serv 1992; 11(3):429-445.

16. Laurell AEC. Políticas de salud en pugna: aseguramiento frente a sistemas universales públicos. Rev $\mathrm{La}$ tino-Am Enferm 2016; 24:e2668.

17. Jaramillo Molina ME. Lo que el neoliberalismo nos dejó [Internet]. Nexos; 2018 [acceso 2020 Ag 28]. Disponible en: https://economia.nexos.com.mx/?p=2034.

18. Dirección General de Información en Salud (DGIS) Base de datos de defunciones 1998-2018. Cubos Dinámicos [Internet]. México: Sistema Nacional de Información en Salud (SINAIS); 2018 [acceso 2019 Nov 15]. Disponible en: http://pwidgis03.salud.gob.mx/ cubos/defunciones/seed_98_2018_hist.htm.

19. Consejo Nacional de Población (CONAPO). México en cifras/Proyecciones de la población 2010-2050/Datos de proyecciones/Estimaciones (1990-2010) [Internet]. México: CONAPO; 2014 [acceso 2019 Nov 25]. Disponible en: http://www.conapo.gob.mx/es/CONA$\mathrm{PO} /$ Proyecciones .

20. Instituto Mexicano de la Juventud. Hacia una perspectiva de Juventud [Internet]. Ciudad de México; 2019 [acceso 2020 May 25]. Disponible en: https://www. gob.mx/imjuve/documentos/hacia-una-perspectivade-juventud.

21. Consejo Nacional de Población (CONAPO). Índice de marginación por entidad federativa y municipio [Internet]. 2015 [acceso 2020 Feb 27]. Disponible en: https://www.gob.mx/conapo/documentos/indicede-marginacion-por-entidad-federativa-y-municipio-2015.

22. Arriaga EE. Los años de vida perdidos: su utilización para medir el nivel y el cambio de la mortalidad. Notas Poblaci 1996; 24(63):7-38.

23. Arriaga EE. Measuring and explaining the change in life expectancies. Demography 1984; 21(1):83-96.

24. Boleda M, Arriaga E. América Latina: Mortalidad por accidentes y por violencia contra las personas. Notas Poblaci 2000; 28(70):87-119.

25. Xunta de Galicia. Epidat. Versión 3.1 [Internet]. La Coruña: Xunta de Galicia, OPS; 2006.

26. Instituto Nacional de Estadística y Geografía (INEGI). Encuesta Intercensal [Internet]. 2015 [acceso 2020 May 15]. Disponible en: https://www.inegi.org. $\mathrm{mx} /$ programas/intercensal/2015/.

27. Dirección General de Información en Salud (DGIS). Base de datos de Recursos en Salud. Cubos Dinámi$\cos$ [Internet]. [acceso 2020 May 15]. Disponible en: http://sinerhias.salud.gob.mx/cubos/sinerhias/recursos_2001_2018_sinerhias.htm.

28. Banco Mundial. Esperanza de vida al nacer, total (años) [Internet]. [acceso 2020 May 25]. Disponible en: https://datos.bancomundial.org/indicador/SP.DYN. LE00.IN.
29. Organización Mundial de la Salud (OMS). Estadísticas Sanitarias Mundiales 2014. Datos positivos sobre la esperanza de vida. Comunicado de prensa [Internet]. [acceso 2020 Ene 15]. Disponible en: https://www. who.int/mediacentre/news/releases/2014/world-health-statistics-2014/es/1/.

30. Fuerte-Celis MP, Pérez-Lujan E. ¡La violencia cambia!: Securitización y configuración territorial del crimen organizado en México, 2007-2011. J Lat Am Geography 2017; 16(3):59-81.

31. González-Pérez GJ, Vega-López MG. Homicidio juvenil en México y su impacto en la esperanza de vida masculina: variaciones geográficas y factores asociados. Salud Colect 2019; 15:e1712.

32. Rivera-Domarco JA, Hernández-Ávila M, AguilarSalinas CA, Vadillo-Ortega F, Murayama-Rendón C. Obesidad en México. Recomendaciones para una política de estado. Ciudad de México: UNAM; 2018.

33. Instituto Nacional de Salud Pública. Encuesta Nacional de Salud y Nutrición de Medio Camino 2016 (ENSANUT 2016). Informe final de resultados [Internet]. Ciudad de México: Secretaría de Salud; 2016.

34. Gobierno de México. Seguro Popular. Infocancer [Internet]. [acceso $2020 \mathrm{Ag}$ 27]. Disponible en: https:// www.infocancer.org. $\mathrm{mx} /$ ? $\mathrm{c}=$ pacientes- $y$-medicos $\& a=$ seguro-popular.

35. Abramo L, Cecchini S, Ullmann H. Enfrentar las desigualdades en salud en América Latina: el rol de la protección social. Cien Saude Colet 2020: 25(5):15871598.

36. Organización Panamericana de la Salud (OPS). Indicadores básicos 2019: Tendencias de la salud en las Américas. Washington, D.C.: OPS; 2019.

Artículo presentado en 31/05/2020

Aprobado en 09/04/2021

Versión final presentada en 11/04/2021

Editores jefes: Romeu Gomes, Antônio Augusto Moura da Silva 
